Intersections

Canadian Journal of Music

Revue canadienne de musique
Intersections CANADAN TOURAA OP NUSIC

\title{
Claude Abromont. 2019. Guide de l'analyse musicale. Dijon (Fr) : Éditions Universitaires de Dijon, 457 p. ISBN 978-2-36441-318-4 (couverture souple)
}

\section{Brice Tissier}

Volume 38, numéro 1-2, 2018

URI : https://id.erudit.org/iderudit/1071679ar

DOI : https://doi.org/10.7202/1071679ar

Aller au sommaire du numéro

Éditeur(s)

Canadian University Music Society / Société de musique des universités canadiennes

ISSN

1911-0146 (imprimé)

1918-512X (numérique)

Découvrir la revue

Citer ce compte rendu

Tissier, B. (2018). Compte rendu de [Claude Abromont. 2019. Guide de l'analyse musicale. Dijon (Fr) : Éditions Universitaires de Dijon, 457 p. ISBN

978-2-36441-318-4 (couverture souple)]. Intersections, 38(1-2), 163-167.

https://doi.org/10.7202/1071679ar

Copyright @ Canadian University Music Society / Société de musique des universités canadiennes, 2020
Ce document est protégé par la loi sur le droit d'auteur. L'utilisation des services d'Érudit (y compris la reproduction) est assujettie à sa politique d'utilisation que vous pouvez consulter en ligne.

https://apropos.erudit.org/fr/usagers/politique-dutilisation/ 


\section{BOOK REVIEW / RECENSION}

Claude Abromont. 2019. Guide de l'analyse musicale. Dijon (Fr) : Éditions Universitaires de Dijon, 457 p. ISBN 978-2-36441-318-4 (couverture souple).

Paru en mai 2019 aux Éditions Universitaires de Dijon, le Guide de l'analyse musicale s'inscrit dans la continuité des précédents guides élaborés par Claude Abromont aux éditions Fayard (en collaboration avec Eugène de Montalembert) : le Guide de la théorie de la musique (2001), le Guide des formes (2010), et le Guide des genres de la musique occidentale (2010).

Outre cette continuité éditoriale, la position professionnelle de l'auteur mérite d'être soulignée quant à la problématique de la présente entreprise: actuellement professeur titulaire de l'une des classes supérieures d'Analyse Musicale au Conservatoire Supérieur de Musique et de Danse de Paris (CNSMDP), Claude Abromont est ainsi le premier non-compositeur à occuper ce poste $^{1}$. Musicologue de formation, il a d'abord été professeur d'analyse pour les étudiants instrumentistes, avant de mettre sur pied, en 2017 au CNSMDP, une classe de «méthodologie de l'analyse». Dans le cadre de celle-ci, il propose un panorama des nombreuses techniques d'analyse existantes, par-delà les époques et les identités géographiques. Le présent Guide s'inscrit donc dans la continuité de cette perspective méthodologique plus globale: dresser - autant que faire se peut - un état des lieux des diverses pratiques analytiques recensées en 2019. Consacré ici à l'une des dimensions les plus «techniques» des disciplines musicologiques, ce Guide souhaite bien entendu s'inscrire également dans la continuité de la bibliographie existante sur le sujet, en proposant une synthèse qui vise l'accomplissement d'un triple objectif

D'abord, il s'agit évaluer d'un œil critique l'état des connaissances actuelles de l'analyse musicale en fonction des diverses sources - dans l'Avant-propos notamment - afin de cerner les forces et les lacunes en matière de connaissances, et de déterminer les théories et les pratiques les plus prometteuses. L'auteur souhaite par ensuite se démarquer de certaines travaux précédents, à

1 Claude Abromont revient d'ailleurs, dans le chapitre «Analyse de compositeur» qui ouvre la Phase 3: approfondissement de l'ouvrage, sur la tradition d'enseignants compositeurs pour la classe d'analyse du CNSMDP depuis près de soixante-dix ans, avec notamment ses prédécesseurs Olivier Messiaen, Claude Ballif, Betsy Jolas, Alain Louvier et Michaël Lévinas pour ne citer que ces quelques noms. Pour Abromont, il n'existe cependant pas d'expression consacrée afin de définir le statut de l'analyste - compositeur, musicologue, interprète ou mélomane (Abromont 2019, 61). 
commencer par le fameux ouvrage de Ian Bent, Analyse musicale (1980); cette importante référence, qui est le fruit de la notice consacrée par Bent à l'analyse pour le dictionnaire Grove, a posé les bases de la pratique de l'analyste. Ainsi, Abromont revient sur la définition générale de l'analyse musicale donnée par Bent, à savoir: « [qu'elle consiste en] la résolution d'une structure musicale en éléments constitutifs relativement plus simples, et la recherche des fonctions de ces éléments à l'intérieur de cette structure» (Bent 1980, 9). Abromont prône ainsi une vision plus large et plus riche de la discipline, c'est pourquoi il intègre dans son Guide nombre de facteurs et d'approches externes à la partition, à commencer par l'observation préalable, qui serait tout aussi importante que l'application de techniques analytiques académiques ou paramétriques (Abromont 2019, 20):

Comme règle initiale, je propose donc de différer le plus longtemps possible le moment où faire appel à sa compétence théorique devient judicieux - tout particulièrement lorsqu'elle est considérable. Il existe bien d'autres questionnements à privilégier lors de l'inauguration d'une quête analytique.

Abromont insiste enfin sur la nécessité de ne pas se limiter à une approche de «niveau neutre» de l'œuvre - pour reprendre un terme issu de la tripartition sémiologique de Jean-Jacques Nattiez. Il convient d'explorer tant la dimension documentaire telle que le support, les sources (esquisses et correspondances), le contexte ou les travaux déjà publiés, que les possibles exploitations du travail d'analyse, sous forme de publication, ou de projet de médiation (note de programme, conférence ou concert lecture).

L'une des dimensions majeures de cette publication réside par ailleurs dans la volonté d'Abromont de plaider pour une pratique moderne et éclairée de l'analyse musicale, c'est-à-dire plus universelle, à la fois sur le fond par un répertoire limité qui sort des cadres des classes officielles et universitaires, et sur la forme en remettant en question la primauté de l'analyse harmonique et structurelle (Abromont 2019, 37-38):

En exagérant jusqu'à l'absurde le «best-of» des œuvres que privilégient les classes d'analyse, il ne serait pas impossible qu'un étudiant puisse pratiquer l'analyse musicale en étudiant exclusivement des compositions de Bach, de Haydn, de Mozart, de Beethoven, de Schubert, de Schumann, de Brahms et de Schönberg. Et cela aurait des vertus pédagogiques évidentes, des normes se dégageraient sans contradictions flagrantes, tandis qu'un héritage technique commun semblerait couler de source.

Une telle approche rassurante serait pourtant à double tranchant. D'une grande efficacité au moment de définir les notions à connaître, assez pratique pour la réussite aux épreuves d'examen, elle s'effectuerait au prix d'un renoncement terriblement précoce à la découverte de l'immense variété des propos, des esthétiques, des cultures, voire des époques, certaines pouvant être très reculées. 
[...] Il est important de résister à l'envie de se cantonner à "l'autoroute» des extraits des «grands chefs-d'œuvre», le «mainstream»si fréquemment germanique, pour aller plutôt de travers, flâner, humer, s'interroger. Et on se sent alors souvent démuni, manquant d'outils adaptés devant tant d'univers surprenants. Tâtonner, tâtonner, l'immersion dans le réel est à ce prix.

Fondamentalement, ce nouveau Guide se veut avant tout un ouvrage pédagogique et utilitaire pour un étudiant ou pratiquant de l'analyse: le plan de l'ouvrage suit un cheminement de travail chronologique nécessaire pour l'étude d'une œuvre dans toutes ses dimensions (Abromont 2019, 14):

Si tous les ouvrages [consacrés à l'analyse musicale] s'adressent aux analystes, aucun n'est véritablement destiné à l'analyste en œuvre. Un ensemble des pratiques efficaces est certes proposé à celui qui veut analyser, des méthodes sont présentées, mais sans s'adresser directement à l'analyste - ou au futur analyste - qui souhaite se repérer dans l'immense champ des possibles.

Cinq chapitres principaux structurent l'ouvrage, suivant une logique d'approche de la partition musicale: 1. la phase de découverte, 2. la phase de documentation, 3. la phase d'approfondissement, 4. la phase de synthèse et 5. la phase de restitution. Chacune de ces phases est scrupuleusement détaillée. La troisième phase dite d'approfondissement est la plus étoffée de l'ouvrage (287 pages). Elle propose un panorama des nombreuses pratiques analytiques, à savoir, successivement: l'analyse de compositeur ${ }^{2}$, du contrepoint, de l'espace, de la forme, génétique, de l'harmonie (la plus détaillée, en 9 sous-parties), implicative, de l'interprétation, des intervalles, inventive, des modes, motivique, de la musique électroacoustique, de la musique non écrite, narratologique, paradigmatique, phraséologique, prolongationnelle, rhétorique, du rythme, schenkérienne, sémantique, sérielle, du style, en terme d'identité sexuelle, de la texture, thématique et réduction analytique. Une mention spéciale mérite donc d'être faite quant à la présentation et la synthèse riche et variée proposée ici de techniques analytiques dont l'auteur présente les forces, les faiblesses et les principaux usages.

$\mathrm{Du}$ fait de cette synthèse, on pourrait peut-être regretter l'absence d'un nombre conséquent d'applications et d'exemples concrets ou complets de ces méthodes dans le corps de l'ouvrage. Pour ne donner qu'un exemple: le chapitre consacré à l'analyse paradigmatique - d'après les travaux de Nicolas Ruwet — ne reprend que le Geisserlied étudié par Ruwet $(1972,116)$.

Pour pallier, Abromont se défend cependant de vouloir développer l'application de chaque technique, non sans arguments: éviter dans ce Guide une lourdeur et un nombre de pages qui serait répulsif et contre-productif (Abromont 2019,14 ) en renvoyant le plus possible vers les ouvrages spécialisés. Par ailleurs, les médias actuels offrant de nouveaux horizons, Abromont fait le choix «d'externaliser» ses propres analyses par le biais de deux canaux sur la plateforme

2 Voir note infrapaginale $n^{\circ} 1$. 
YouTube, dont les liens sont indiqués dans l'ouvrage (Abromont 2019, 14-15). Le premier canal contient les démonstrations et exemples musicaux présentés dans ce Guide (référencés dans l'ouvrage par des pictogrammes); le second est accessible aux chercheurs et pédagogues désireux d'éclairer tel ou tel point du Guide. L'ambition de l'auteur est ainsi de ne pas «figer» ce livre dans sa date de publication (2019), mais au contraire d'en faire un outil vivant et prospère, ouvert à toutes les participations et contributions.

Internet permet par ailleurs la mise en corrélation de la musique avec la partition ou des images - dans la limite évidente du respect du droit d'auteur mais également le commentaire/analyse simultané(e). Parmi les analyses déjà présentes sur internet, et mettant en application les éléments du livre, citons trois réalisations remarquables de l'auteur lui-même:

- La schématisation globale du Kammerkonzert d'Alban Berg 3 mettant en évidence les structures internes mais aussi métrotechtoniques de l'œuvre;

- Le Prélude à l'après-midi d'un faune de Claude Debussy ${ }^{4}$ avec confrontation en suivi des différentes analyses publiées - notamment en ce qui concerne le découpage de la pièce;

- La synchronisation de la réduction par Czerny avec l'enregistrement de l'étude $n^{\circ} 1$ de Chopin 5 .

$S$ 'il fallait émettre quelques réserves quant à cet ouvrage, on pourrait peut-être évoquer la question des quelques compositeurs échappant aux codes analytiques académiques, et dont la spécificité suscite tant l'attraction des jeunes analystes que la réticence chronique des professeurs d'analyse. Citons par exemple Scriabine et Hindemith, dont les langages harmoniques, singuliers, nécessiteraient un traitement particulier. Abromont en fait mention succinctement (2019, 135-138). Toutefois, force est de constater que la singularité des compositeurs depuis la fin du XIXème siècle, sans compter la «non-contemporanéité des contemporains » prônée par Carl Dahlhaus, impose un frein indéniable et inéluctable à la capacité de synthèse de l'analyse. L'auteur renvoie pour chaque cas exposé aux références bibliographiques spécialisées.

Probablement que l'un des manques les plus marquants de ce Guide de l'analyse musicale de Claude Abromont concerne l'utilisation de l'ordinateur comme outil d'analyse 6 . De plus en plus, le recours à l'outil informatique permet en effet la compréhension d'œuvres complexes, ou de systèmes d'écriture complexes. Par exemple, l'œuvre de Iannis Xenakis, conçue à l'aide de lois mathématiques et de programmes informatiques, ne saurait désormais plus - en partie du moins - être étudiée sans le recours à l'ordinateur. De même, les œuvres conçues à partir de programmes de synthèse, de Composition Assistée par Ordinateur $(\mathrm{CAO})$ ou destinées à une exécution en «temps réel» ne

3 https://www.youtube.com/watch?v=rJ5cGRFOW14 (consulté en septembre 2019).

4 https://www.youtube.com/watch?v=-uolYjeP8dM (consulté en septembre 2019).

5 https://www.youtube.com/watch?v=Ks4oT-4wObY (consulté en septembre 2019).

6 Plusieurs pages sont néanmoins consacrées à certains logiciels d'analyse assistée par ordinateur, comme SonicVisualiser, permettant la confrontation d'enregistrements musicaux, et donc une étude de l'interprétation (Abromont 2019, 194-200). 
pourront être appréhendées globalement sans le recours de la technologie; les esquisses des compositeurs ne reflètent malheureusement pas ces considérations. L'analyste doit-il donc s'imposer une formation aux nouvelles technologies - et de constantes mises à jour ! - pour pratiquer sa discipline? L'analyse musicale et la très grande majorité du répertoire ne sont, heureusement, pas encore soumises à ces considérations.

Pour conclure, il convient de mettre en avant la facilité de lecture du Guide de l'analyse musicale de Claude Abromont, et la multiplicité des approches que permet l'ouvrage: une approche pédagogique et chronologique par la lecture linéaire du livre; ainsi qu'une approche thématique et sélective des différents chapitres. L'index, particulièrement détaillé en fin d'ouvrage et signalant les œuvres analysées dans le corps du texte, renforce également cet aspect utilitaire. Connecté aux analyses désormais publiées en ligne et annoncées chroniquement sur les réseaux sociaux (Facebook), ce Guide se révèle être un outil vivant qui ne fera que s'enrichir dans les années à venir.

\section{RÉFÉRENCES}

Bent, Ian. 1980 [trad. française 1998]. L’analyse musicale. Paris: Éditions Main d'œuvre.

Ruwet, Nicolas. 1972. Langage, musique, poésie. Paris: Éditions du Seuil.

BRICE TISSIER

\section{BIOGRAPHY}

Titulaire de quatre prix du CNSM de Paris, Brice Tissier est agrégé de musique et docteur en musicologie de l'Université de Paris-Sorbonne et de l'Université de Montréal (PhD). Il a été directeur musical du Chœur Grégorien de Paris de 2001 et 2008. Il est l'auteur de plusieurs articles pour des revues françaises, canadiennes et tchèques, consacrés à l'étude de l'œuvre de Pierre Boulez, Karlheinz Stockhausen et Philippe Manoury, la restitution du chant grégorien, et l'octobasse de Jean-Baptiste Vuillaume. Il enseigne l'analyse au Conservatoire à Rayonnement Régional et au Pôle Supérieur (Isdat) de Toulouse, ainsi que l'histoire de la musique du XX ${ }^{\text {ème }}$ siècle à l'Université de Toulouse Jean-Jaurès. 